\title{
Comment on "Lower Limb Muscle Size After Anterior Cruciate Ligament Injury: A Systematic Review and Meta-Analysis"
}

\author{
Han Zhang ${ }^{1} \cdot$ Guanhong Chen ${ }^{1}$ (I) \\ Accepted: 27 September 2021 / Published online: 21 October 2021 \\ (c) The Author(s), under exclusive licence to Springer Nature Switzerland AG 2021
}

\section{Dear Editor,}

We carefully read the article "Lower limb muscle size after anterior cruciate ligament injury: a systematic review and meta-analysis" by Dutaillis et al. [1]. The authors explored the effect of anterior cruciate ligament injury on muscle size in the lower limbs and found that the quadriceps muscle of the injured limb after an anterior cruciate ligament injury is smaller than the equivalent muscle of the uninjured limb. When semitendinosus/gracilis tendon grafts were used for reconstruction, the harvested muscle showed a smaller muscle size than the uninjured limb. The methodology used by the authors involved a meta-analysis. Although the authors' analytical methods were scientific, we think there are some problems in this paper that need to be improved.

1. 49 articles were included in the systematic review, and data from 37 of them were used for the meta-analysis. However, we did not find a table that provided details of the 49 articles included in the authors' review. The characteristics of the articles included in a meta-analysis are generally presented in a paper as a table.

2. The authors used a modified version of the Downs and Black Checklist in the quality assessment of the literature included in the article. However, this method of literature quality assessment has not been recognized. The Cochrane risk of bias assessment tool is more widely recommended for the quality assessment of articles. In

An author's reply to this letter is available at https://doi.org/10. 1007/s40279-021-01577-9.

This comment refers to the article available online at https://doi. org/10.1007/s40279-020-01419-0.

Guanhong Chen

zbzbzlm@163.com

1 Department of Joint Surgery, Shanxian Central Hospital, Heze 274000, China this review, the authors only evaluated the publication bias of meta-analyses involving more than ten studies, which was not rigorous. We think the authors should have used Egger's test to evaluate the publication bias of all meta-analyses in this article.

3. It is also not rigorous to use the random-effects model indiscriminately in a meta-analysis. The random-effects model and the fixed-effects model should be selected according to the magnitude of heterogeneity. As a general rule, heterogeneity between studies is tested by the $I^{2}$ statistic with $I^{2} \geq 50 \%$ indicating heterogeneity. If significant heterogeneity exists, a random-effects model is used; if no significant heterogeneity is found, a fixedeffects model is used [2].

Admittedly, the authors' research is scientifically rigorous. However, we believe that if the above issues are improved, the conclusions drawn from this analysis will be more instructive for clinical work.

\section{Declarations}

Funding No sources of funding were used to assist in the preparation of this letter.

Conflict of Interest Han Zhang and Guanhong Chen declare that they have no conflicts of interest with the content of this letter.

\section{References}

1. Dutaillis B, Maniar N, Opar DA, Hickey JT, Timmins RG. Lower limb muscle size after anterior cruciate ligament injury: a systematic review and meta-analysis. Sports Med. 2021;51(6):1209-26. https://doi.org/10.1007/s40279-020-01419-0.

2. Sun P, Bi M, Chen Z. Meta-analysis should be carried out objectively and rigorously. J Clin Anesth. 2019;56:6-16. https://doi. org/10.1016/j.jclinane.2018.12.055. 